\title{
Comparative Clinical and Angiographic Analysis of the Initial Efficacy and Long-term Follow-up of Wiktor Stent Implantation with Conventional Balloon Angioplasty
}

\author{
Ender Semiz, MD, Oktay Sancaktar, MD, \\ Selim Yalçinkaya, MD, Hilmi Ege, MD, \\ and Necmi DEĞER, MD
}

\section{SuMMARY}

This study was retrospectively designed to examine if the Wiktor stent, a balloon-expandable tantalum coil stent, provides a more favorable procedural and long-term clinical and angiographic outcome than does conventional coronary balloon angioplasty (POBA). From April 1995 to April 1996, we implanted 56 Wiktor stents in 46 lesions (LAD: 23, RCA: 16, CX: 7) in 42 patients (average age $53 \pm 10$ years). Coronary lesions from the stent group were matched with similar lesions of another 42 POBA patients whose characteristics were identical to the Wiktor group. Revascularization indications in the Wiktor and POBA groups, respectively, were recent myocardial infarction (RMI) $(45 \%, 40 \%)$, unstable $(31 \%, 39 \%)$ and stable $(24 \%, 21 \%)$ angina pectoris. $7 \%$ of the stents and $17 \%$ of the POBA balloons were less than $3 \mathrm{~mm}$ in diameter $(p>0.05)$. Procedural success was significantly greater in the Wiktor group than in the POBA group (100\% vs. 92\%, $p<0.05$ ). Neither major cardiac event (death, CABG, acute myocardial infarction) nor (sub)acute occlusion was encountered in the Wiktor group during the hospitalization period and 1 month follow-up. There was 1 urgent $\mathrm{CABG}$ and 4 subacute occlusions in the POBA group. Control angiography at 8 months was performed in patients of both groups, of whom some were symptomatic at long-term follow-up or completely event free for 8 months. Angiographic restenosis $>50 \%$ diameter stenosis) occurred in $25 \%$ of the Wiktor patients and in $43 \%$ of those in the POBA group $(p<0.05)$. For an 18 month clinical follow-up, $91 \%$ of the patients in the Wiktor group were asymptomatic and without ischemia in radionuclide imaging $(\mathrm{RI})$, whereas $79 \%$ of the POBA patients were angina-free and $74 \%$ were without ischemia in the RI study.

In conclusion, Wiktor stent implantation, with no major cardiac event or subacute occlusion, provides a more favorable procedural and long-term clinical and angiographic outcome than does conventional POBA. (Jpn Heart J 1997; 38: 625-635)

From Department of Cardiology, Akdeniz University School of Medicine, Antalya, Turkey

Address for correspondance: Ender Semiz, MD, Akdeniz University School of Medicine, Department of Cardiology, 07060, Antalya, Turkey.

Received for publication December 16, 1996.

Accepted March 17, 1997. 
Key words: Coronary stents, Wiktor stent, Angioplasty, Coronary, Restenosis

$\mathrm{T}$ recent ycars, coronary stenting has been developed to overcome abrupt vessel closure, and late stenosis after conventional percutaneous coronary balloon angioplasty (POBA). Randomized STRESS and BENESTENT trials clearly demonstrated improved clinical outcomes ${ }^{1)}$ and reduced angiographic restenosis ${ }^{1,2)}$ when compared with POBA for elective single-vessel coronary revascularization.

This study reports the procedural results of Wiktor stenting and conventional POBA, together with clinical and angiographic outcomes at 8 month follow-up. Clinical and radionuclide imaging (RI) follow-up of both groups every 5 months for a period of 18 months are also reported in this study.

\section{METHODS}

Study patients: Between April 1995 and April 1996, 56 Wiktor stents were implanted in 46 lesions in 42 patients (1.33 stents per patient). A total of 568 patients referred for POBA were screened for entry into the study. 236 of 568, who underwent a control coronary angiography at the $8^{\text {th }}$ month were matched to a group of 42 patients, to serve as a control group. These patients had clinical and lesion characteristics similar to those in the Wiktor group. The mean age of the Wiktor group was $53 \pm 10$ years (range 38 to 72 ), and 35 cases $(83 \%)$ were male. The POBA group, of whom $33(79 \%)$ were male, had a mean age of $55 \pm 9$ years (range 34 to 77). Revascularization indications in the Wiktor and POBA

Table 1. Patient Characteristics

\begin{tabular}{lccc}
\hline & Wiktor Group $(n=42)$ & POBA Group $(n=42)$ & $p$ Valuc \\
\hline Male (\%) & $35(83)$ & $33(79)$ & NS \\
Age (years) & $53 \pm 10($ range $38-72)$ & $55 \pm 9$ (range 34-77) & NS \\
Stable angina pectoris (\%) & $10(24)$ & $12(29)$ & NS \\
Unstable angina pectoris (\%) & $13(31)$ & $16(38)$ & NS \\
Recent MI (\%) & $19(45)$ & $14(33)$ & NS \\
Prior POBA (\%) & $6(14)$ & $4(10)$ & NS \\
Prior MI (\%) & $7(17)$ & $6(14)$ & NS \\
Prior CABG $(\%)$ & $2(5)$ & 0 & NS \\
Coronary risk factors (\%) & $34(81)$ & $30(71)$ & NS \\
$\quad$ Smoking history & $6(14)$ & $9(21)$ & NS \\
Diabetes & $25(60)$ & $27(64)$ & NS \\
Cholesterol $\geq 200 \mathrm{mg} / \mathrm{d} l$ & $16(38)$ & $20(48)$ & NS \\
$\quad$ Hypertension & $23(55)$ & $19(45)$ & NS \\
Family history & & & \\
\hline
\end{tabular}

Data presented are mean values $\pm \mathrm{SD}$ or number $(\%)$ of patients. $\mathrm{MI}=$ myocardial infarction; $\mathrm{POBA}=$ balloon angioplasty; $\mathrm{CABG}=$ coronary artery bypass grafting; $\mathrm{NS}=$ non-significant. 
groups, respectively, were recent myocardial infarction (RMI) $(45 \%, 40 \%)$, and unstable $(31 \%, 39 \%)$ and stable $(24 \%, 21 \%)$ angina pectoris. Patient characteristics are outlined in Table I.

Study protocol: All of the patients had a $>70 \%$ stenosis in their native vessels. Coronary flow distal to the lesion was classified before the procedure according to the Thrombolysis in Myocardial Infarction Trial (TIMI) criteria. ${ }^{3)}$ Just before both procedures, all patients received a bolus of $10,000 \mathrm{IU}$ heparin intravenously and subsequently $5,000 \mathrm{IU}$ as required to maintain the activated clotting time $>300$ seconds throughout the procedure. Intracoronary injections of nitroglycerine were administered to minimize any possible spasm and to achieve maximal vasodilatation. The study protocol was approved by the Committee for Clinical Investigation at Akdeniz University School of Medicine and written informed consent was obtained from all patients.

The Wiktor balloon-expandable intracoronary stent was used in this study. It consists of a single strand of radiopaque tantalum wire wrapped around an angioplasty balloon (coil stent) which offers marked flexibility and thus conformability with the vessel curvature. A 0.014 inch coronary guidewire is used to deliver the stent balloon system via an 8-Fr. guiding catheter. The current prototype of the Wiktor stent measures $16 \mathrm{~mm}$ in length, which is the only available size in the market. Except for one, all of the Wiktor stent group procedures were planned. Patients were premedicated with aspirin (300 $\mathrm{mg} /$ day) and ticlopidine (250 mg PO bid) for 48 hours before implantation. Following predilatation of the target lesions, 56 stents were implanted in 46 lesions in 42 patients. High-pressure inflations (Swiss-Kiss) (mean 15 2 atm., range 13-22 atm) were used to optimize stent expansion after initial deployment of the stent. Following successful stent implantation, patients were given ticlopidine and aspirin for 1 month and indefinitely, respectively.

Over-the-wire or monorail balloons 20-mm in length, which were available in the cathetcrization laboratory during the POBA procedure, were used. All patients reccived aspirin ( $300 \mathrm{mg}$ chewed) and diltiazem $(60 \mathrm{mg}$ ) in addition to nitroglycerin infusion before the POBA procedure. A 0.014 inch coronary guidewire and a 7-Fr. guiding catheter were used as the delivery system. The balloon sizes were matched to the reference diameter with a ratio of 1:1 (inflated balloon diameter: artery diameter). Following POBA, oral isosorbide mononitrate $(40 \mathrm{mg}$ bid) was added to aspirin $(300 \mathrm{mg} /$ day $)$ and diltiazem $(60$ $\mathrm{mg}$ tid) for a period of at least 6 months.

Control coronary angiography was performed in both groups following an 8 month follow-up. Redilatation was attempted in patients with restenosis. Clinical information for an 18 month (range 14-22) follow-up period was obtained through an interview during outpatient clinic visits or from the patient or family 
by telephone. End points in this analysis were death from all causes, nonfatal myocardial infarction, treatment with repeat POBA or coronary artery bypass surgery and recurrent angina (Canadian Cardiovascular Society (CCS) Classification). Ischemia in RI studies has also been investigated every 5 months for a mean follow-up period of 18 months.

Study end points and definitions: Acute (in the first day following the procedure) or subacute (in the first month following the procedure) thrombosis, repeat intervention, coronary artery bypass surgery ( $\mathrm{CABG})$, acute myocardial infarction (AMT), or death were the primary clinical end points of the study. The secondary end point was restenosis rate. A new $Q$ wave or elevation of myocardial enzyme levels to more than twice the upper limit of normal, or both, were accepted as evidence of AMI. Procedural success for stenting and POBA was defined as successful deployment of the stent and successful dilatation of the target lesion, respectively, in the absence of a major cardiac event (MACEDeath, AMI, CABG). A < 10\% residual diameter stenosis following final deployment of the stent was used to define angiographic success for stent implantation. A suboptimal result was defined as post-procedural residual diameter stenosis of 10-50\%. Decrease in percent diameter at the stenotic site of less than 50\% was accepted as angiographic success for POBA. Restenosis for both groups was defined as having a $>50 \%$ stenosis on angiograms at the 8 month follow-up. Any event at the 18 month follow-up was categorized as AMI, CABG, death, target vessel revascularization (TVR) and cerebrovascular accident.

Measurements and statistics: The lesion diameter was assessed visually during diastole using the image in a single "worst" view. The proximal part of the vessel nearest the initial occlusion was used to measure the reference vessel diameter. The same measurements were repeated in the same views during follow-up angiograms. Continuous variables are expressed as the mean value $\pm \mathrm{SD}$. Categorical variables were presented as absolute numbers (\%). Comparisons between groups were made by use of Student $t$-tests for continuous data and chi-square analysis for categorical data. A $p$ value $<0.05$ was considered significant.

\section{Results}

Angiographic lesion characteristics of both groups are listed in Table II. Of 46 lesions in the Wiktor group, 23 were in the left anterior descending coronary artery (LAD), 16 in the right coronary artery (RCA), and the remaining 7 in the left circumflex coronary artery (CX). Of 42 lesions in the POBA group, 19 were in the $\mathrm{LAD}, 17$ in the RCA and 6 in the CX. $65 \%$ of the lesions before predilatation in the Wiktor group and $62 \%$ of the lesions before conventional POBA were types $B_{2}$ and $C(p>0.05)$, respectively, according to the American 
Table II. Angiographical Data

\begin{tabular}{|c|c|c|c|}
\hline & Wiktor Group $(n=42)$ & POBA Group $(n=42)$ & $p$ Value \\
\hline Number of lesions $(\%)$ & 46 & 42 & NS \\
\hline LAD & $23(50)$ & $19(45)$ & NS \\
\hline RCA & $16(35)$ & $17(41)$ & NS \\
\hline $\mathrm{CX}$ & $7(15)$ & $6(14)$ & NS \\
\hline \multicolumn{4}{|l|}{ Lesion morphology $\left\langle^{*}\right\rangle(\%)$} \\
\hline Type A & $5(11)$ & $6(14)$ & NS \\
\hline Type $B_{1}$ & $11(24)$ & $10(24)$ & NS \\
\hline Type $B_{2}$ & $24(52)$ & $21(50)$ & NS \\
\hline Type C & $6(13)$ & $5(12)$ & NS \\
\hline$\%$ diameter stenosis & 84 & 86 & NS \\
\hline \multicolumn{4}{|l|}{ TIMI flow before intervention (\%) } \\
\hline TIMI 0 & $1(2)$ & 0 & NS \\
\hline TIMI 1 & $3(7)$ & $1(2)$ & NS \\
\hline TIMI 2 & $2(4)$ & $1(2)$ & NS \\
\hline TIMI 3 & $40(87)$ & $40(95)$ & NS \\
\hline \multicolumn{4}{|l|}{ Type of dissection before stent (\%) } \\
\hline Type A & $4(9)$ & $\cdots$ & - \\
\hline Type B & $6(13)$ & - & - \\
\hline Type C & $1(2)$ & - & - \\
\hline Type D & 0 & - & - \\
\hline Type E & $1(2)$ & - & - \\
\hline Type F & 0 & - & - \\
\hline Thrombus before intervention (\%) & $22(48)$ & $15(36)$ & NS \\
\hline \multicolumn{4}{|l|}{ Reference vessel diameter $(\%)$} \\
\hline$<3.0 \mathrm{~mm}$ & $3(7)$ & $7(17)$ & NS \\
\hline$\geq 3.0 \mathrm{~mm}$ & $43(93)$ & $35(83)$ & NS \\
\hline \multicolumn{4}{|l|}{ Number of diseased vessels $(\%)$} \\
\hline I vessel disease & $37(88)$ & $33(79)$ & NS \\
\hline 2 vessel disease & $5(12)$ & $8(19)$ & NS \\
\hline 3 vessel disease & 0 & $1(2)$ & NS \\
\hline
\end{tabular}

Data presented are number $(\%)$ of lesions, unless otherwise indicated. $\mathrm{LAD}=$ left anterior descending artery; $\mathrm{RCA}=$ right coronary artery; $\mathrm{CX}=$ circumflex artery; TIMI $=$ thrombolysis in myocardial infarction; NS = nonsignificant.

(*) Modified AHA / ACG lesion classification.

Heart Association/American College of Cardiology (AHA/ACC) Task Force criteria.) Angiographic appearance of intracoronary thrombus was present in $48 \%$ of the lesions in the Wiktor and $36 \%$ of the lesions in the POBA groups $(p>0.05) .7 \%$ of the vessels in the Wiktor and $17 \%$ of the vessels in the POBA group were less than $3 \mathrm{~mm}$ in diameter, respectively $(p>0.05)$. Angiographic success as defined by $<10 \%$ residual diameter stenosis was achieved in all of the cases (42 of 42) in the Wiktor group. Procedural success was significantly lower in the POBA group $(100 \%$ vs. $93 \%, p<0.05)$. In two cases in the stent group, there was a tendency towards accordion shape deformation of the stent, which was solved by proceeding with lower profile balloons. Except for this, no difficulty was encountered in any patient in recrossing the Wiktor stent with a high pressure balloon for post dilatation. No complication such as MACE or (sub)acute occlu- 
sion occurred in the Wiktor group. One urgent CABG and 4 subacute occlusions $(p<0.05)$ were encountered in the POBA group. Hematoma formation requiring blood transfusion at the femoral puncture site was encountered in two of the Wiktor group but in none of the POBA patients. Pseudoaneurysm formation at the femoral puncture site was seen in only 1 case of the stent group, with no other complication requiring surgical repair. The patients of both groups were discharged post procedure after a mean period of 1.5 days. Procedural results are summarized in Table III.

Except for two patients with acute myocardial infarction in both groups, all patients remained event free for a period of 8 months. Symptomatic patients at long-term follow-up or patients who completed their event free period of 8 months underwent control coronary angiography. Angiographic restenosis (>50\% diameter stenosis) rate was $25 \%(10 / 40)$ in 40 of the $42(95 \%)$ Wiktor

Table III. Procedural and Post-procedural Characteristics

\begin{tabular}{|c|c|c|c|}
\hline & Wiktor Group $(n=42)$ & POBA Group $(n=42)$ & $p$ Value \\
\hline \multicolumn{4}{|l|}{ Indication for revascularization (\%) } \\
\hline Elective & $41 / 46(89)$ & $42 / 42(100)$ & NS \\
\hline Restenosis following prior POBA & $5 / 46(11)$ & - & - \\
\hline Suboptimal result & 0 & - & $\cdots$ \\
\hline Bail-out & $1 / 46(2)$ & - & - \\
\hline Procedural success $(\%)$ & $56 / 56(100)$ & $39 / 42(93)$ & $<0.05$ \\
\hline \multicolumn{4}{|l|}{ Major cardiac event (\%) } \\
\hline Death & 0 & 0 & - \\
\hline Acute MI & 0 & 0 & - \\
\hline Urgent CABG & 0 & $1(2)$ & NS \\
\hline Acute / subacute closure (\%) & 0 & $4(10)$ & $<0.05$ \\
\hline Number of stents / patients (\%) & $56 / 42$ & - & - \\
\hline Single stent & $31(74)$ & - & - \\
\hline 2 stents & $9(2 \mathrm{l})$ & 一 & - \\
\hline 3 stents and over & $2(5)$ & - & - \\
\hline Blood transfusion (\%) & $2(5)$ & 0 & NS \\
\hline Surgical repair (\%) & $1(2)$ & 0 & NS \\
\hline
\end{tabular}

Data presented are number (\%) of patients, unless otherwise indicated. POBA = balloon angioplasty; $\mathrm{MI}=$ myocardial infarction; $\mathrm{CABG}=$ coronary artery bypass grafting; $\mathrm{NS}$ = nonsignificant.

Table IV. Functional Class 18 Months after Intervention

\begin{tabular}{cccc}
\hline & Wiktor Group $(n=42)$ & POBA Group $(n=42)$ & $p$ Value \\
\hline CCS Functional Class (\%) & & & \\
No angina & $38(91)$ & $33(79)$ & NS \\
I & $2(5)$ & $3(7)$ & NS \\
II & $1(2)$ & $5(12)$ & $<0.05$ \\
III & $1(2)$ & $1(2)$ & NS \\
IV & 0 & 0 & - \\
\hline
\end{tabular}

Data presented are number $(\%)$ of patients; CCS = Canadian Cardiovascular Society; NS = nonsignificant. 
group patients. The remaining 2 patients did not agree to control angiography. The restenosis rate in the POBA group was $43 \%(18 / 42)$. The difference between restenosis rates was significant $(p<0.05)$. TVR by POBA in the Wiktor group was performed successfully in 8 patients $(20 \%)$ without any MACE. Two of the restenotic cases refused intervention. TVR in the POBA group was successful in 13 patients $(31 \%)$ with no MACE. Three patients in this group underwent elective $\mathrm{CABG}$, which was significantly different $(p<0.05)$ when compared with the frequency of elective CABG (none) in the Wiktor group. The remaining 2 patients did not receive intervention because their restenotic vessels were very thin in caliber. One of them also had a highly proximal LAD lesion, which is known to have a high restenosis rate.

Angina class at the 18th month follow-up, with $91 \%$ of Wiktor and $79 \%$ of POBA patients remaining angina-free, is shown in Table IV. When compared with 1 patient in the Wiktor group, five patients in the POBA group were suffering from CCS class II angina. This was a statistically significant difference $(p<0.05)$. There were 2 AMI, 6 CABG procedures, and 1 death in the POBA group but none in the Wiktor group. The difference between the frequency of CABG in the groups $(21 \%$ vs. $0 \%)$ was significant $(p<0.05)$. No ischemia in any of the 38 angina-free Wiktor patients $(91 \%)$ was detected in the RI studies performed every 5 months during this period of 18 months. The remaining 4 pa-

Table V. Clinical End Points at 8 and 18 Months

\begin{tabular}{|c|c|c|c|}
\hline & Wiktor Group $(n=42)$ & POBA Group $(n=42)$ & $p$ Value \\
\hline \multicolumn{4}{|l|}{8 month follow-up $(\%)$} \\
\hline Control angiography & $40 / 42(98)$ & $42 / 42(100)$ & NS \\
\hline Restenosis & $10 / 40(25)$ & $18 / 42(43)$ & $<0.05$ \\
\hline TVR & $8 / 40(20)$ & $13 / 42(31)$ & $<0.05$ \\
\hline Q wave MI & $1 / 42(2)$ & $2 / 42(5)$ & NS \\
\hline Non-Q wave MI & $1 / 42(2)$ & 0 & NS \\
\hline $\mathrm{CABG}$ & 0 & $3(7)$ & $<0.05$ \\
\hline Death & 0 & 0 & 一 \\
\hline CVA & 0 & 0 & - \\
\hline \multicolumn{4}{|l|}{18 month follow-up $(\%)$} \\
\hline Number of patients & $42 / 42(100)$ & $42 / 42(100)$ & NS \\
\hline $\operatorname{rePOBA}\left(^{*}\right)$ & 0 & 0 & - \\
\hline Q wave MI & $1 / 42(2)$ & $4(10)$ & NS \\
\hline Non-Q wave MI & $1 / 42(2)$ & 0 & NS \\
\hline CABG & 0 & $9(21)$ & $<0.05$ \\
\hline Death & 0 & $1(2)$ & NS \\
\hline CVA & 0 & 0 & - \\
\hline Any event at 18 month follow-up (\%) & $10 / 42(24)$ & $27 / 42(64)$ & $<0.05$ \\
\hline
\end{tabular}

Data presented are number $(\%)$ of patients; TVR $=$ target vessel revascularization; $\mathrm{MI}=$ myocardial infarction; $\mathrm{CABG}=$ coronary artery bypass grafting; $\mathrm{CVA}=$ cerebrovascular accident; rePOBA = repeat balloon angioplasty; Any event = AMI, CABG, TVR, CVA or death; NS = nonsignificant.

$\left.{ }^{*}\right)$ rePOBA performed between 9 th and 18 th month follow-up. 
tients with angina (9\%) had mild myocardial perfusion defects in RI that were thought unlikely to be due to restenosis. Thus, they were considered not important enough to perform control angiography. Nine patients in the POBA group (21\%) were experiencing angina, resulting in a significant difference $(p<0.05)$ when compared with the angina frequency of the stent group. A mild to moderate degree of ischemia in RI study was reported in $26 \%$ of the POBA patients $(11 / 42)$, of whom 9 were experiencing angina. This result was also comparable $(p<0.05)$ with the frequency of ischemia (mild) detected in the stent group (9\%) (not shown in the table). Any event at the 18 month follow-up in the POBA group was higher than in the Wiktor group ( $64 \%$ vs. $24 \%, p<0.05$ ). Clinical end points at 8 and 18 months are outlined in Table $\mathrm{V}$.

\section{Discussion}

Abrupt vessel closure in the early period of conventional POBA and restenosis during late follow-up are the main problems compromising its overall efficacy ${ }^{5-10)}$ Abrupt closure is associated with significant morbidity and mortality, and is thought to be the result of a combination of factors such as dissection, spasm and thrombus formation. It occurs in 2 to $10 \%$ of cases. Smooth muscle cell proliferation at the site of dilation causes extensive neointimal thickening and late restenosis, occurring in 20 to $40 \%$ of cases, and may necessitate repeat POBA or CABG. ${ }^{11,12 j}$

Intracoronary stent implantation has been developed to overcome these two major limitations of POBA. A number of different stents are available at present with their specific designs, compositions and delivery systems. ${ }^{13-16)}$ The Wiktor stent is a balloon-expandable stent made of a single, loose tantalum wire 0.125 $\mathrm{mm}$ in diameter and has a helical coil structure. The potential for corrosion and stress fracture is minimized by its sinusoidal helix design. Stents can be distinguished by their type of delivery system, composition and configuration. Despite all coronary stents currently evaluated being metallic, each has a different degree of radiopacity and radial compliance. The Wiktor stent (Medtronic Inc., Minneapolis, MN), originally designed by Dominik Wiktor, is a balloon-expandable tantalum coil stent with advantages of high radiopacity and flexibility. ${ }^{17}$ Lowmetallic surface area $(8.8 \%)$ in combination with its design and electrochemical properties of tantalum may protect against thrombus formation.

In this study, we compared the initial and long-term clinical and angiographic outcomes of successful Wiktor stent implantation and POBA. In matched patients with similar lesion location and severity, Wiktor stenting was associated with a significantly larger immediate vessel lumen, that was preserved during the 8 month angiographic follow-up. The success rate of elective implan- 
tation of all types of intracoronary stents range from 95 to $100 \%$. Initial experience with elective Wiktor stent implantation in humans with restenosis following POBA revealed a thrombosis rate of $10 \%$ in the acute or subacute phase following implantation. ${ }^{17}$ ) Following coronary stenting, adding ticlopidine (250 mg bid) to postprocedural treatment with aspirin and low molecular weight heparin, French investigators ${ }^{18,19)}$ observed a reduction in the incidence of subacute thrombosis from $10.4 \%$ (145 patients) to $1.3 \%$ (237 patients who received 1 month of subcutaneous low molecular weight heparin (SLMWH), 1.7\% (523 patients who received 2 weeks of heparin) and $1.8 \%$ (491 patients who received 1 week of heparin). Full antiplatelet therapy (ticlopidine and aspirin) without additional SLMWH with high-pressure stent implantation has become routine clinical practice at present. In our study, we used both ticlopidine and aspirin for 48 hours before implantation and for 1 month and indefinitely following the procedure, respectively. There was no acute or subacute closure.

The results of the present study reveal that the frequency of angiographic restenosis at the 8 month follow-up point tends to be higher in patients with successful POBA than in those with successful Wiktor stent implantation (43\% vs. $25 \%, p<0.05$ ). The comprehensive analysis of the 8 month follow-up of Wiktor stenting and POBA suggests that the scaffolding effect of the stent may lead to less lumen loss, that is a larger late lumen, than achieved with POBA alone. In contrast to the favorable results observed with the Palmaz-Schatz stent (Benestent I, Stress I, and Start $)^{1,2,20,21)}$ restenosis trials, primary Wiktor stenting of the right coronary artery in a pilot study did not reveal a clinical or angiographic advantage over conventional POBA with 6 month restenosis rates of $47.5 \%$ and $35 \%$, respectively. ${ }^{22)}$ In this pilot study, ${ }^{22)}$ angioplasty (occasionally up to 8 bars) within the stent had always been repeated after Wiktor stent implantation to attempt full stent expansion. Lack of high pressure ( $\geq 12$ bars) postdilatation might be the reason for the high rate of restenosis in that pilot study. The European Wiktor Stent Study Group reported a $>50 \%$ arterial diameter narrowing in $27 \%$ of patients with threatened or acute closure complicating POBA. ${ }^{23)}$ The incidence of restenosis greater than $50 \%$ diameter stenosis for the Wiktor stent was reported to be $30 \%,{ }^{1724)}$ which seems to be higher than ours. Our results at the 8 month follow-up with a restenosis rate of $25 \%$ demonstrate an acceptable rate of stent restenosis compared with the results of the other studies described above.

In conclusion, the present study shows that the Wiktor stent implantation with no MACE provides more favorable short- and long-term clinical and angiographic outcomes than does conventional POBA. Angiographic outcome at the 8 month follow-up is acceptable. Thirty-eight of 42 patients (91\%) were asymptomatic during a mean period of 18 months. RI studies with exercise stress testing done every 5 months also revcaled no ischemia in angina-free Wiktor 
patients during this follow-up period.

Study limitations: Our study has several limitations. First, this is not a randomized study. Retrospective analysis of the data is the second limitation. The third limitation is that relatively small patient groups were studied. Another limitation is that the current study was conducted in a single center. Qualitative coronary angiography was performed in this study since we do not have quantitative coronary angiography in our laboratory.

\section{REFERENCES}

1. Serruys PW, de Jaegere P, Kiemeneij F, et al. A comparison of balloon-expandable-stent implantation with balloon angioplasty in patients with coronary artery disease. N Engl J Med 1994; 331: 489-95.

2. Fischman DL, Leon MB, Baim DS, et al. A randomized comparison of coronary-stent placement and balloon angioplasty in the treatment of coronary artery disease. N Engl J Med 1994; 331: 496-501.

3. Buchwald $A$, Unterberg $C$, Werner $G$, et al. Initial clinical results with the Wiktor stent: a new balloon-expandable coronary stent. Clin Cardiol 1991; 14: 374

4. The TIMI Study Group. The Thrombolysis in Myocardial Infarction (TIMI) trial. N Engl J Med 1985; 312: 932-6.

5. Ryan TJ, Faxon DP, Gunnar RM, et al. Guidelines for percutaneous transluminal coronary angioplasty: a report of the American College of Cardiology / American Heart Association Task Force on assessment of diagnostic and therapeutic cardiovascular procedures. Circulation 1988; 78: 486-502.

6. Tuzcu EM, Simpfendorfer $C$, Dorosti $\mathrm{K}$, et al. Changing patterns in percutaneous transluminal coronary angioplasty. Am Heart J 1989; 117: 1374-7.

7. Frierson JH, Dimas AP, Franco I, et al. Angioplasty of the proximal left anterior descending coronary artery: Initial success and long-term follow-up. J Am Coll Gardiol 1992; 19: 745-51.

8. Detre K, Holubkov R, Kelsey S, et al. Percutaneous transluminal angioplasty in 1985-1986 and 1977-1981. The National Heart, Lung, and Blood Institute Registry. N Engl J Med 1988; 318: 265.

9. Leimgruber P, Roubin GS, Hollnan J, et al. Restenosis after successful coronary angioplasty in patients with single-vessel disease. Circulation 1986; 73: 710.

10. MERCATOR Study Group. Does the new angiotensin converting enzyme inhibitor cilazapril prevent restenosis after percutaneous transluminal coronary angioplasty? The results of the Mercator study: a multi-center randonized double-blind placebo-controlled trial. Circulation 1992; 86: 100.

11. Simpfendorfer C. Acute coronary occlusion after percutaneous transluminal coronary angioplasty. Cleve Clin J Med 1988; 55: 429.

12. Hollman J, Goormastic M, Simpfendorfer C, Raymond R, Whitlow P. Comparison of coronary angioplasty: multivessel versus single vessel angioplasty -- long-term data. J Am Goll Cardiol 1990; 15: $205 \mathrm{~A}$.

13. O'Keefe JH, Rutherford BD, McConahay DR, et al. Multivessel coronary angioplasty from 1980 to 1989: procedural results and long-term outcome. J Am Coll Cardiol 1990; 16: 1097-102.

14. Sigwart U, Puel J, Mirkovitch V, Joffre F, Kappenberger L. Intravascular stents to prevent occlusion and restenosis after transluminal angioplasty. $\mathrm{N}$ Engl J Med 1987; 316: 701.

15. Roubin GS, Cannon AD, Agrawal SK, et al. Intracoronary stenting for acute and threatened closure complicating percutaneous transluminal coronary angioplasty. Circulation 1992; 85: 916-27.

16. Schatz RA, Baim DS, Leon M, et al. Clinical experience with the Palmaz-Schatz coronary stent: initial results of a multicenter study. Circulation 1991; 83: 148.

17. de Jaegere PD, Serruys PW, Bertrand M, et al. Wiktor stent implantation in patients with restenosis following balloon angioplasty of a native coronary artery. Am J Cardiol 1992; 69: 598.

18. Morice MC, Bourdonnec C, Valeix B, et al. Coronary stenting without coumadin. Phase II [abstract]. Eur Heart J 1994; 15: 536.

19. Morice MC, Bourdonnec C, Lefevre $\mathrm{T}$, et al. Coronary stenting without coumadin. Phase III [ab- 
stract]. Circulation 1994; 90 Suppl I: I-125.

20. Macaya G, Serruys PW, Suryapranata $H$, et al. One year clinical follow-up of the Benestent trial [abstract]. J Am Coll Cardiol 1995; 25: 374A.

21. Betriu A, Serra A, Masotti M, et al. The Spanish trial: are national randomized trials a necessary evil? J Intervent Cardiol 1994; 7: 347-53.

22. Eeckhout E, Stauffer JC, Vogt P, Debbas N, Kappenberger L, Goy JJ. Comparison of elective Wiktor stent placement with conventional balloon angioplasty for new-onset lesions of the right coronary artery. Am Heart J 1996; 132: 263-8.

23. Vrolix M, Piessens J, for the European Wiktor Stent Study Group. Usefulness of the Wiktor stent for treatment of threatened or acute closure complicating coronary angioplasty. Am J Cardiol 1994; 73: $737-41$.

24. Serruys $\mathrm{P}$, de Jaegere $\mathrm{P}$, Bertrand $\mathrm{M}$, et al. Morphologic change in coronary artery stenosis with the Medtronic Wiktor stent: initial results from the core laboratory for quantitative angiography. Cathet Cardiovasc Diagn 1991; 24: 237. 